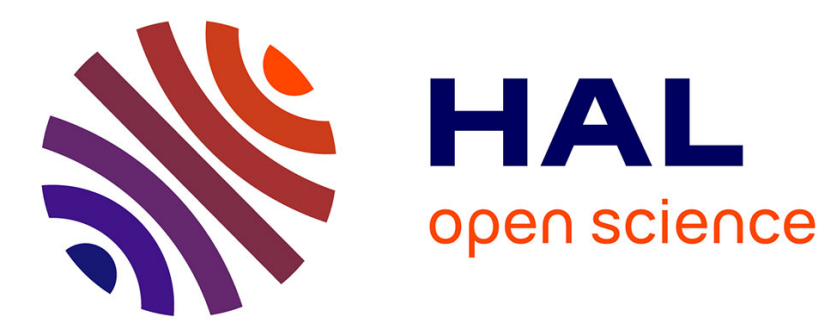

\title{
TRANSFERTS D'ÉNERGIE ENTRE ÉTATS EXCITÉS
}

André Ricard

\section{To cite this version:}

André Ricard. TRANSFERTS D'ÉNERGIE ENTRE ÉTATS EXCITÉS. Journal de Physique Colloques, 1977, 38 (C3), pp.C3-239-C3-247. 10.1051/jphyscol:1977326 . jpa-00217114

\section{HAL Id: jpa-00217114 https://hal.science/jpa-00217114}

Submitted on 1 Jan 1977

HAL is a multi-disciplinary open access archive for the deposit and dissemination of scientific research documents, whether they are published or not. The documents may come from teaching and research institutions in France or abroad, or from public or private research centers.
L'archive ouverte pluridisciplinaire HAL, est destinée au dépôt et à la diffusion de documents scientifiques de niveau recherche, publiés ou non, émanant des établissements d'enseignement et de recherche français ou étrangers, des laboratoires publics ou privés. 


\title{
TRANSFERTS D'ÉNERGIE ENTRE ÉTATS EXCITÉS
}

\author{
A. RICARD \\ Laboratoire de Physique des Plasmas $(*)$, \\ Bâtiment 212, Université Paris XI, Centre d'Orsay, 91405 Orsay, France
}

\begin{abstract}
Résumé. - Les transferts d'énergie entre états excités concernent tout aussi bien la physique des lasers à gaz que la chimie des plasmas. On trouve deux grandes catégories : chimie-ionisation et chimie-excitation. Une distinction est faite entre transferts non réactifs : $\mathrm{A}^{*}+\mathrm{BC} \rightarrow \mathrm{A}+\mathrm{BC}^{+}+\mathrm{e}$ (effet Penning) ou $\mathrm{A}+\mathrm{BC}^{*}$ et transferts réactifs : $\underline{A}^{*}+\mathrm{BC} \rightarrow \mathrm{AB}^{+}+\mathrm{C}+\mathrm{e}$ (ré-arrangement) ou $\mathrm{AB}^{*}+\mathrm{C}$.

Dans le cadre des réactions de chimie-ionisation, on indique l'efficacité relative de l'effet Penning, du ré-arrangement et de la dissociation suivant les travaux de West et al. (1975) et le rôle de la température du gaz d'après Lindinger et al. (1974).

En ce qui concerne les réactions de chimie-excitation non réactives, on mentionne les travaux récents sur les transferts $\underline{\mathrm{Ar}^{*}}+\mathrm{N}_{2}$ qui ont permis de préciser le rôle de la température du gaz et le rapport de branchement pour l'excitation des états $\mathrm{N}_{2}\left(\mathrm{C}^{3} \Pi_{\mathrm{u}}, \mathrm{B}^{3} \Pi_{\mathrm{g}}\right)$. L'action des niveaux résonnants d'argon dans des réactions $\operatorname{Ar}\left({ }^{3} \mathrm{P}_{1},{ }^{1} \mathbf{P}_{1}\right)+\mathrm{BC}{ }^{k} \rightarrow \mathrm{Ar}+\mathrm{BC}^{*}\left(\mathrm{ou} \mathrm{B}^{*}+\mathrm{C}\right.$ ) est analysée pour $\mathrm{BC}=\mathrm{H}_{2}, \mathrm{~N}_{2}, \mathrm{O}_{2}, \mathrm{CO}, \mathrm{NO}$ et $\mathrm{C}_{2} \mathrm{H}_{4}$. Les valeurs expérimentales du coefficient de collisions $k$ sont comparées à celles calculées suivant la théorie de Watanabe-Katsuura (1967).

Les transferts réactifs sont efficaces avec les molécules halogénées $\mathrm{RX}$ où $\mathrm{X}=\mathrm{F}, \mathrm{Cl}$ et $\mathrm{Br}$. Une étude des rapports de branchement pour la formation des excimers $\mathrm{AX}^{*}$ est présentée à partir des résultats obtenus par Gundel et al. (1976) pour les mélanges $\mathrm{Ar}^{*}+\mathrm{RX}$ et par Velazco et al. (1976) pour les mélanges $\left(\mathrm{Kr}^{*}, \underline{\mathrm{Xe}^{*}}\right)+\mathrm{RF}$. Une interprétation est donnée à partir de l'interaction entre le potentiel d'entrée $\left(\mathrm{A}^{*}+\mathrm{RX}\right)$ et la courbe de potentiel coulombien $\left(\mathrm{A}^{+}+\mathrm{RX}^{-}\right)$. Les transferts réactifs $N\left({ }^{2} D\right)+B C$ étudiés par Lin-Kaufman (1971) sont également indiqués.

Enfin, certains transferts dans l'azote pur qui participent à la relaxation globale d'états excités de l'azote sont donnés, à savoir la désexcitation des niveaux $N_{2}\left(B^{3} \Pi_{g}, A^{3} \Sigma_{u}^{+}, E^{3} \Sigma_{g}^{+}, a^{1} \Pi_{g}\right)$.
\end{abstract}

\footnotetext{
Abstract. - The transfer of energy between excited states is important for gas lasers as well as for plasma chemistry. There are two main processes : chemiionisation and chemiexcitation. One distinguishes two processes, non-reactive process : $\mathrm{A}^{*}+\mathrm{BC} \rightarrow \mathrm{A}+\mathbf{B C}^{+}+\mathbf{e}$ (Penning effect), and reactive process : $A^{*}+B C \rightarrow \mathrm{AB}^{+}+\mathrm{C}+\mathrm{e}$ (rearrangement) or $\mathrm{AB}^{*}+\mathrm{C}$.

For chemiionisation, the relative efficiency of the Penning effect, of rearrangement and dissociation is given according to West et al. (1975). The role of the gas temperature is discussed according to Lindinger et al. (1974).

As for non-reactive chemiexcitation reactions recent works on $\underline{A r}^{*}+N_{2}$ transfers is summarised which clarify the role of gas temperature and branching ratio in the excitation of the states $\mathrm{N}_{2}\left(\mathrm{C}^{3} \Pi_{\mathrm{u}}, \mathrm{B}^{3} \Pi_{\mathrm{g}}\right)$. The role of argon resonance states in the reactions $\operatorname{Ar}\left({ }^{3} \mathrm{P}_{1},{ }^{1} \mathrm{P}_{1}\right)+\mathrm{BC} \stackrel{\mathrm{k}}{\rightarrow} \mathrm{Ar}+\mathrm{BC}$ (or $\mathrm{B}^{*}+\mathrm{C}$ ) is analysed for $\mathrm{BC}=\mathrm{H}_{2}, \mathrm{~N}_{2}, \mathrm{O}_{2}, \mathrm{CO}$, NO and $\mathrm{C}_{2} \mathrm{H}_{4}$. Experimental values of the collision coefficient $k$ are compared to those calculated from the theory of Watanabe and Katsuura (1967).

Reactive transfers are efficient with halogen containing molecules $\mathrm{RX}$, where $\mathrm{X}=\mathrm{F}, \mathrm{Cl}$ and $\mathrm{Br}$. A study of branching ratios for the formation of $\mathrm{AX}^{*}$ excimers is presented from results obtained by Gundel et al. (1976) in Ar* $+\mathrm{BC}$ mixtures and by Velazco et al. (1976) in ( $\left.\mathrm{Kr}^{*}, \mathrm{Xe}^{*}\right)+\mathrm{RF}$. An interpretation is given based on the interaction between the inlet potential $\left(A^{*}+R X\right)$ and the Coulomb potential $\left(\mathrm{A}^{+}+\mathrm{RX}^{-}\right)$. The reactive transfers $\mathrm{N}\left({ }^{2} \mathrm{D}\right)+\mathrm{BC}$ studied by Lin-Kaufman (1971) are also given.

Finally, some transfers in pure nitrogen that participate in the global relaxation of the excited states of that molecule are given. This concerns the levels $\mathrm{N}_{2}\left(\mathrm{~B}^{3} \Pi_{\mathrm{g}}, \mathrm{A}^{3} \Sigma_{\mathrm{u}}^{+}, \mathrm{E}^{3} \Sigma_{\mathrm{g}}^{+}, \mathrm{a}^{1} \Pi_{\mathrm{g}}\right)$.
}

(*) Laboratoire Associé au C.N.R.S. 
1. Introduction. - Les transferts d'énergie entre états excités sont à l'origine des mécanismes d'inversion de population et des réactions chimiques dans les plasmas hors d'équilibre. Ils concernent la physique des lasers à gaz et la chimie des plasmas. Le principe de ces transferts est le suivant : une décharge électrique dans un gaz rare produit l'excitation des états métastables et ces états métastables transfèrent leur énergie sur un deuxième élément gazeux qui se trouve excitê, dissocié ou ionisé. Ainsi, dans les mélanges argonazote, les atomes métastables d'argon excitent l'azote. L'émission laser des bandes moléculaires de l'azote, consécutive à ce transfert, est actuellement très étudiée. Du côté de la chimie des plasmas, on produit des espèces d'azote excitées qui jouent un rôle actif dans des réactions de synthèse, notamment avec l'oxygène. Cette double application : lasers et chimie des plasmas s'est encore trouvée renforcée avec l'étude récente des excimers. Les transferts considérés sont cette fois-ci réactifs. Les atomes métastables $\mathrm{A}^{*}$ et les molécules $\mathrm{BC}$ forment des excimers $(\mathrm{AB})^{*}$ suivant la réaction :

$$
\underline{A}^{*}+\mathrm{BC} \rightarrow(\mathrm{AB})^{*}+\mathrm{C} .
$$

Cette réaction à caractère chimique est très efficace. Nous décrivons les transferts d'énergie entre états excités en les partageant en deux grandes catégories :

A) Réactions de chimie-ionisation caractérisées par :

- Effet Penning

$$
\underline{\mathrm{A}}^{*}+\mathrm{BC} \rightarrow \mathrm{A}+(\mathrm{BC})^{+}+\mathrm{e} .
$$

- Ionisation associative

$$
\underline{A}^{*}+B C \rightarrow(A B C)^{+}+e .
$$

- Ionisation avec ré-arrangement

$$
\mathrm{A}^{*}+\mathrm{BC} \rightarrow(\mathrm{AB})^{+}+\mathrm{C}+\mathrm{e} \text {. }
$$

- Ionisation dissociative

$$
\underline{\mathrm{A}}^{*}+\mathrm{BC} \rightarrow \mathrm{A}+\mathrm{B}^{+}+\mathrm{C}+\mathrm{e} .
$$

B) Réaction de chimie-excitation

- Transfert non réactif

$$
\underline{A}^{*}+\mathrm{BC} \rightarrow \mathrm{A}+\mathrm{BC}^{*} \text {. }
$$

- Transfert réactif

$$
\mathrm{A}^{*}+\mathrm{BC} \rightarrow(\mathrm{AB})^{*}+\mathrm{C} .
$$

- Transfert dissociatif

$$
\underline{A}^{*}+B C \rightarrow A+B^{*}+C .
$$

Les réactions de chimie-ionisation ont surtout été étudiées avec les atomes métastables de l'hélium. Ces espèces sont en effet porteurs d'une énergie potentielle suffisante pour ioniser la plupart des gaz moléculaires. L'effet Penning est un mécanisme efficace et très sélectif qui produit des ions $(\mathrm{BC})^{+}$dans un état électronique et vibrationnel bien défini. Les réactions de chimie-excitation sont beaucoup moins sélectives mais peuvent être efficaces surtout dans les transferts réactifs et dissociatifs. Ces réactions se produisent essentiellement avec les atomes métastables et radiatifs des gaz rares lourds, à bas potentiel d'excitation. Nous nous intéressons ensuite aux transferts entre états excités de l'azote et en particulier aux phénomènes de relaxation des états électroniques excités.

2. Mécanismes de chimie-ionisation. - L'effet Penning, schématisé par la réaction 2 , est le mécanisme le plus important. Les réactions avec l'hélium suivant :

$$
\mathrm{He}\left(2^{3} \mathrm{~S}\right)+\mathrm{BC} \stackrel{k^{+}}{\rightarrow} \mathrm{He}+\mathrm{BC}^{+}+\mathrm{e},
$$

ont été étudiées avec $\mathrm{BC}=\mathrm{H}_{2}, \mathrm{~N}_{2}, \mathrm{O}_{2}, \mathrm{Cl}_{2}, \mathrm{CH}_{4}$, $\mathrm{NH}_{3}, \mathrm{H}_{2} \mathrm{O}, \mathrm{HCl}, \mathrm{HBr}, \mathrm{CO}, \mathrm{CO}_{2}, \mathrm{NO}, \mathrm{N}_{2} \mathrm{O}$ et $\mathrm{SF}_{6}$ et les valeurs du coefficient de collisions $k^{+}=\sigma^{+} \frac{6}{v}$ $\left(\sigma^{+}\right.$section efficace d'ionisation Penning, $\bar{v}$ vitesse relative des espèces en interaction) ont été déterminées. Une synthèse des résultats obtenus est donnée en réf. [1].

Des résultats récents ont été obtenus par West et al. [2] pour l'ensemble des réactions de chimieionisation (2) à (5) mettant en jeu les atomes métastables de l'hélium, du néon, de l'argon et du krypton. Les rapports de branchement pour chacune des réactions (2) à (5) ont été déterminés, les résultats obtenus avec $\mathrm{He}\left(2^{3} \mathrm{~S}\right)$ et $\mathrm{Ne}\left({ }^{3} \mathrm{P}_{0,2}\right)$ sont indiqués dans le tableau I. L'ionisation associative de NO, suivant la réaction (3), augmente avec les gaz rares lourds : $0,7 \%$ de $\mathrm{NeNO}^{+}, 19 \%$ de $\mathrm{ArNO}^{+}$et $36 \%$ de $\mathrm{K}_{\mathrm{r}} \mathrm{NO}^{+}$. Le mécanisme de ré-arrangement (réaction 4) se produit essentiellement avec $\mathrm{H}_{2}$.

L'efficacité de ces réactions dépend de la température du milieu. Ainsi, il est montré par Lindinger et al. [5] que le coefficient d'ionisation $k^{+}$des réactions $\mathrm{He}\left(2^{3} \mathrm{~S}\right)+\mathrm{BC}$ croît avec la température d'autant plus vite que les molécules $\mathrm{BC}$ sont moins polarisables. Pour $300<T<900 \mathrm{~K}$, les coefficients $k^{+}$peuvent s'exprimer suivant l'équation du type Arrkénius :

$$
k^{+}(T)=1,9 \times 10^{-9} \exp \left(-\frac{E_{\mathrm{A}}}{0,057}-\frac{E_{\mathrm{A}}}{k T}\right)
$$

où $k^{+}$est en $\mathrm{cm}^{3} \mathrm{~s}^{-1}$ et l'énergie d'activation $E_{\mathrm{A}} \mathrm{en} \mathrm{eV}$. Les valeurs de $E_{\mathrm{A}}$ trouvées pour différentes molécules $\mathrm{BC}$ sont indiquées dans le tableau II.

Les transferts Penning s'interprètent suivant un mécanisme d'échange au cours duquel un électron de la molécule $\mathrm{BC}$ prend la place vacante de l'électron 1s (cas du métastable de l'hélium) tandis que l'électron 2 s est éjecté. Si l'orbitale externe de BC est du type $\sigma$ (cas de $\mathrm{N}_{2}, \mathrm{CO}$ ), le circuit d'entrée du complexe moléculaire $\mathrm{He}\left(2^{3} \mathrm{~S}\right)+\mathrm{BC}$ est répulsif. Si, par contre, la molécule $\mathrm{BC}$ possède une orbitale externe du type $\pi$ (cas de $\mathrm{O}_{2}, \mathrm{NO}$ ), le circuit d'entrée est attractif. Avec un circuit d'entrée répulsif, le transfert Penning est immédiat : les deux atomes B et C peuvent être considérés comme immobiles. On dit que la transition 
TABLEAU I

$\underline{\mathrm{A}}^{*}+\mathrm{BC} \rightarrow$ produits [2] (les références sont indiquées entre parenthèses après les valeurs des sections efficaces $\sigma$ )

\begin{tabular}{|c|c|c|c|c|c|c|}
\hline \multirow{3}{*}{ Molécules BC } & \multicolumn{3}{|c|}{$\underline{A}^{*}=\operatorname{He}\left(2^{3} S\right)$} & \multicolumn{3}{|c|}{$\underline{\mathrm{A}}^{*}=\mathrm{Ne}\left({ }^{3} \mathrm{P}_{0,2}\right)$} \\
\hline & Ions formés & $\begin{array}{c}\text { Rapport de } \\
\text { branchement }\end{array}$ & $\begin{array}{c}\sigma \text { totale } \\
\left(10^{-16} \mathrm{~cm}^{2}\right)\end{array}$ & Ions formés & $\begin{array}{l}\text { Rapport de } \\
\text { branchement }\end{array}$ & $\begin{array}{c}\sigma \text { totale } \\
\left(10^{-16} \mathrm{~cm}^{2}\right)\end{array}$ \\
\hline & - & - & - & - & - & - \\
\hline $\mathrm{H}_{2}$ & $\mid \begin{array}{l}\mathbf{H}_{2}^{+} \\
\mathrm{HeH}^{+} \\
\mathbf{H}^{+} \\
\mathrm{HeH}_{2}^{+}\end{array}$ & $\mid \begin{array}{l}0,88 \\
0,081 \\
0,021 \\
0,015\end{array}$ & $3,7(3)$ & $\mid \begin{array}{l}\mathrm{H}_{2}^{+} \\
\mathrm{NeH}^{+} \\
- \\
\mathrm{NeH}_{2}^{+}\end{array}$ & $\mid \begin{array}{l}0,78 \\
0,22 \\
-\end{array}$ & $2,65(2)$ \\
\hline $\mathrm{N}_{2}$ & & & & $\mid \begin{array}{l}\mathrm{N}_{2}^{+} \\
\mathrm{NeN}_{2}^{+}\end{array}$ & $\mid \begin{array}{l}0,941 \\
0,059\end{array}$ & $10,4(2)$ \\
\hline $\mathrm{O}_{2}$ & $\mid \begin{array}{l}\mathrm{O}_{2}^{+} \\
\mathrm{O}^{+}\end{array}$ & $\mid \begin{array}{l}0,94 \\
0,06\end{array}$ & $29,4(4)$ & $\mid \begin{array}{l}\mathrm{O}_{2}^{+} \\
\mathrm{NeO}_{2}^{+}\end{array}$ & $\mid \begin{array}{l}0,997 \\
0,003\end{array}$ & $25,5(2)$ \\
\hline $\mathrm{CO}$ & & & & $\mid \begin{array}{l}\mathrm{CO}^{+} \\
\mathrm{NeCO}^{+}\end{array}$ & $\mid \begin{array}{l}0,925 \\
0,075\end{array}$ & $11,2(2)$ \\
\hline $\mathrm{CO}_{2}$ & $\mid \begin{array}{l}\mathrm{CO}_{2}^{+} \\
\mathrm{CO}^{+} \\
\mathrm{O}^{+}\end{array}$ & $\mid \begin{array}{l}0,69 \\
0,045 \\
0,27\end{array}$ & $63,1(4)$ & $\mid \begin{array}{l}\mathrm{CO}_{2}^{+} \\
\mathrm{NeCO}_{2}^{+}\end{array}$ & $\mid \begin{array}{l}0,994 \\
0,0055\end{array}$ & $37,1(2)$ \\
\hline No & $\mid \begin{array}{l}\mathrm{NO}^{+} \\
\mathrm{HeNO}^{+}\end{array}$ & 1 & $38,1(4)$ & $\mid \begin{array}{l}\mathrm{NO}^{+} \\
\mathrm{NeNO}^{+}\end{array}$ & $\mid \begin{array}{l}0,993 \\
0,007\end{array}$ & $21,4(2)$ \\
\hline $\mathrm{N}_{2} \mathrm{O}$ & $\mid \begin{array}{l}\mathrm{N}_{2} \mathrm{O}^{+} \\
\mathrm{NO}^{+} \\
\mathrm{O}^{+}\end{array}$ & $\left|\begin{array}{l}0,47 \\
0,51 \\
0,024\end{array}\right|$ & $37,7(4)$ & $\mid \begin{array}{l}\mathrm{N}_{2} \mathrm{O}^{+} \\
\mathrm{NO}^{+} \\
\mathrm{O}^{+} \\
\mathrm{NeN}_{2} \mathrm{O}^{+}\end{array}$ & $\mid \begin{array}{l}0,76 \\
0,17 \\
0,063 \\
0,007\end{array}$ & $24,8(2)$ \\
\hline
\end{tabular}

TABLEaU II

$$
\begin{gathered}
\mathrm{He}\left(2^{3} \mathrm{~S}\right)+\mathrm{BC} \stackrel{k^{+}(T)}{\rightarrow} \text { produits } \\
k^{+}(T)=1,9 \times 10^{-9} \exp \left(-\frac{E_{\mathrm{A}}}{0,057}-\frac{E_{\mathrm{A}}}{k T}\right)[5]
\end{gathered}
$$

$\begin{array}{ccccccccccccc}\begin{array}{c}\text { Molécules } \\ \mathrm{BC}\end{array} & \mathrm{H}_{2} & \mathrm{~N}_{2} & \mathrm{O}_{2} & \mathrm{CH}_{4} & \mathrm{NH}_{3} & \mathrm{CO} & \mathrm{CO}_{2} & \mathrm{NO} & \mathrm{N}_{2} \mathrm{O} & \mathrm{C}_{2} \mathrm{H}_{6} & \mathrm{C}_{3} \mathrm{H}_{8} & \mathrm{C}_{4} \mathrm{H}_{10} \\ \begin{array}{c}\text { Energie } \\ \text { d'activation }\end{array} & - & - & - & - & - & - & - & - & - & - & - & - \\ E_{\mathrm{A}}\left(10^{-3} \mathrm{eV}\right) & 72 & 59 & 36 & 47 & 12 & 53 & 19 & 37 & 28 & 37 & 34 & 28\end{array}$

$\mathrm{BC} \rightarrow \mathrm{BC}^{+}$(réaction 2) est verticale et qu'elle s'effectue suivant le principe de Franck-Condon.

Lorsque le circuit d'entrée est attractif, il y a formation d'un complexe : le transfert est long et perd de sa sélectivité. Par contre, l'interaction est puissante. Un potentiel d'entrée $\mathrm{He}\left(2^{3} S\right)+B C$ attractif peut en effet couper plusieurs courbes potentielles de sortie $\mathrm{He}+\mathbf{B C}^{+*}$ répulsives, augmentant ainsi les possibilités de transfert d'où sa probabilité.

Le cas des transferts $\mathrm{He}\left(2^{3} \mathrm{~S}\right)+\mathrm{NO}$ est analysé par Coxon et al. [6]. Il y a partage moitié-moitié entre un transfert Penning et une dissociation non ionisante de la molécule NO, la réaction s'écrit :

$$
\begin{array}{r}
\mathrm{He}\left(2^{3} \mathrm{~S}\right)+\mathrm{NO} \longrightarrow \mathrm{He}+\mathrm{NO}^{+}(\mathrm{A}, \mathrm{X})+\mathrm{e} \\
\longrightarrow \mathrm{He}+\left\{\begin{array}{l}
\mathrm{N}^{*}+\mathrm{O} \\
\mathrm{O}^{*}+\mathrm{N} .
\end{array}\right.
\end{array}
$$

La partie du transfert constituée par l'effet Penning serait analogue au transfert $\mathrm{He}\left(2^{3} \mathrm{~S}\right)+\mathrm{N}_{2}$ : circuit d'entrée répulsif, transfert à longue distance et immédiat.

La partie de dissociation non ionisante serait analogue au transfert $\mathrm{He}\left(2^{3} \mathrm{~S}\right)+\mathrm{O}_{2}$ : circuit d'entrée attractif, formation de complexes et fort couplage avec des circuits de sortie répulsifs conduisant à la dissociation.

3. Mécanismes de chimie-excitation. - Les mécanismes de chimie-excitation se distinguent de ceux de chimie-ionisation par le fait essentiel que l'énergie du métastable $A^{*}$ est insuffisante pour ioniser la molécule BC. $\mathrm{C}^{\top}$ est dans ce domaine que les lasers à transferts se situent et que la chimie des plasmas peut prendre un développement considérable puisque des 
espèces métastables d'énergie inférieure à $10 \mathrm{eV}$ peuvent produire des transferts réactifs.

3.1 TRANSFERTS DISSOCIATIFS ET NON RÉACTIFS. Ces processus correspondent aux réactions 7 et 6 . Les réactions $\mathrm{Ar}^{*}+\mathrm{BC}$ avec $\mathrm{BC}=\mathrm{H}_{2}, \mathrm{~N}_{2}, \mathrm{O}_{2}, \mathrm{CO}$, $\mathrm{NO}, \mathrm{Cl}_{2}, \mathrm{H}_{2} \mathrm{O}, \widetilde{\mathrm{HCl}}, \mathrm{H}_{2} \mathrm{O}, \mathrm{N}_{2} \mathrm{O}, \mathrm{COS}, \mathrm{CS}_{2}, \mathrm{SF}_{6}$ et les réactions $\mathrm{N}_{2}(\mathrm{~A})+\mathrm{BC}$ avec $\mathrm{BC}=\mathrm{H}_{2}, \mathrm{~N}_{2}, \mathrm{O}_{2}, \mathrm{CH}_{4}$, $\mathrm{C}_{2} \mathrm{H}_{2}, \mathrm{C}_{2} \overline{\mathrm{H}_{4}, \mathrm{C}_{2}} \mathrm{H}_{6}, \mathrm{CO}, \mathrm{NO}, \mathrm{HN}_{3}, \mathrm{C}_{2} \mathrm{~N}_{2}, \mathrm{C}_{4} \mathrm{H}_{8}, \mathrm{SO}_{2}$ sont répertoriées dans la référence 1 . Les valeurs des coefficients de collisions et les observations spectroscopiques y sont indiquées en particulier. Depuis, les transferts $\mathrm{Ar}^{*}+\mathrm{N}_{2}$ ont été à nouveau étudiés pour connaître d'une part le rôle de la température sur le coefficient de transfert et d'autre part le rapport de branchement pour les différents états $\mathrm{N}_{2}\left(\mathrm{C}^{3} \Pi_{\mathrm{u}}\right.$ et $\mathrm{B}^{3} \Pi_{\mathrm{g}}$ ) formés à la suite du transfert. En ce qui concerne l'effet de la température, il est montré par Lee-Martin [7] que le coefficient du transfert $\mathrm{Ar}^{*}+\mathrm{N}_{2}$ double lorsque la température croît de 50 à $700 \mathrm{~K}$. Sanders et al. [8] ont constaté qu'en valeurs relatives, le coefficient de transfert passe par un maximum pour $T \sim 1700 \mathrm{~K}$ et décroît légèrement jusqu'à $3500 \mathrm{~K}$, le seuil de la réaction est à $T=580 \mathrm{~K}$.

Le rapport de branchement a été déterminé pour les deux circuits de sortie suivants :

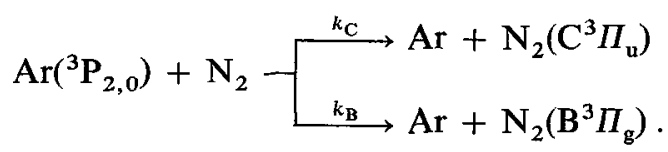

Setser-Stedman [9] donne $k_{\mathrm{B}} / k_{\mathrm{c}}=6 \pm 1$. Ce résultat est utilisé par Hill et al. [10] dans le calcul du bilan d'excitation des lasers $\mathrm{Ar}-\mathrm{N}_{2}$. Il semble que cette valeur soit trop forte et que $k_{\mathrm{B}} / k_{\mathrm{c}} \leqslant 1,3$ suivant Touzeau et al. [11].

Les transferts à partir des niveaux résonnants des gaz rares présentent un intérêt particulier du fait qu'ils se produisent en général à longue distance par une interaction dipôle-dipôle. Ce type d'interaction est décrit par la théorie de Watanabe-Katsuura [12] dans les conditions suivantes :

1) L'interaction est à grande distance; les trajectoires sont rectilignes, les intégrales de recouvrement peuvent être négligées.

2) La molécule $\mathrm{BC}$ a un moment angulaire nul et possède un continuum qui ne présente pas de structures discontinues en photoabsorption. Des sections efficaces de photoabsorption $\sigma_{\mathrm{a}}$ ont été déterminées expérimentalement dans la référence [13] pour NO et 15 hydrocarbures aux longueurs d'ondes

$$
\lambda=1066,6 \AA \text { et } 1048,2 \AA
$$

correspondant aux raies de résonance de l'argon.

3) L'atome résonnant $A^{*}$ est dans l'état $P$.

Le coefficient de collisions calculé s'écrit :

$$
\begin{aligned}
k_{\mathrm{th}}\left(\mathrm{cm}^{3} \mathrm{~s}^{-1}\right)=2,6 \times 10^{-10} & {\left[\mu_{\mathrm{n}}^{2}(\mathrm{a} \cdot \mathrm{u}) \mu_{E_{\mathrm{n}}}^{2}(\mathrm{a} \cdot \mathrm{u})\right]^{2 / 5} \times } \\
\times & {\left[\frac{T(\mathrm{~K})}{M(\mathrm{a} \cdot \mathrm{u})}\right]^{3 / 10} }
\end{aligned}
$$

où (a.u) désigne les unités atomiques.

- $\mu_{\mathrm{n}}^{2}$ est le carré du moment dipolaire du niveau résonnant, on a $\mu_{\mathrm{n}}^{2}=\frac{f_{\mathrm{on}}}{2\left(E_{\mathrm{n}}-E_{\mathrm{o}}\right)}$ avec $E_{\mathrm{n}}, E_{0}$, les énergies des niveaux résonnants $(\mathrm{n})$ et fondamental $(\mathrm{o})$, $f_{\text {on }}$ force d'oscillateur.

$-\mu_{E_{\mathrm{n}}}^{2}=\sigma_{a} \lambda_{\mathrm{no}} /\left(2 \pi a_{\mathrm{o}}\right)^{3}, \lambda_{\mathrm{no}}$ longueur d'onde $\mathrm{du}$ rayonnement de résonance, $a_{\mathrm{o}}$ rayon de Bohr.

- $M$ masse réduite, $T$ température du milieu.

\begin{tabular}{|c|c|c|c|}
\hline Molécules BC & $\begin{array}{l}\text { Molécules excitées } \\
\mathrm{BC}^{*}\left(\text { ou } \mathrm{B}^{*}+\mathrm{C}\right)\end{array}$ & $\begin{array}{l}\text { Coefficients mesurés } k_{3,1} \\
\qquad\left(10^{-11} \mathrm{~cm}^{3} \mathrm{~s}^{-1}\right)\end{array}$ & $\begin{array}{l}\text { Coefficients calculés } k_{3,1}^{\text {th }} \\
\qquad\left(10^{-11} \mathrm{~cm}^{3} \mathrm{~s}^{-1}\right)\end{array}$ \\
\hline - & 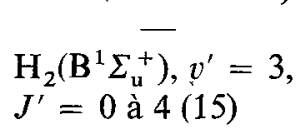 & $\begin{array}{c}- \\
k_{3}=21(14) ; 20(16) \\
k_{1}=22(14) ; 33(16)\end{array}$ & - \\
\hline $\mathrm{N}_{2}$ & $\mathrm{~N}_{2}\left(\mathrm{C}^{3} \Pi_{\mathrm{u}}\right)\left(\mathrm{B}^{3} \Pi_{\mathrm{g}} ?\right)$ & $\begin{aligned} k_{3}= & 1,2(17) ; 0,48(18) \\
& 0,8(14) \\
k_{1}= & 0,74(17) ; 5,4(14)\end{aligned}$ & , \\
\hline $\mathrm{O}_{2}$ & $\begin{array}{l}\mathrm{O}\left(2^{1} \mathrm{~S}\right)+\mathrm{O} \\
\mathrm{O}\left(2^{1} \mathrm{D}\right)+\mathrm{O}\end{array}$ & $\begin{array}{l}k_{3}=9,6(19) ; 0,72(18) \\
k_{1}=11(19)\end{array}$ & \\
\hline $\mathrm{CO}$ & & $\begin{array}{l}k_{3}=4,5(16) \\
k_{1}=8,5(16)\end{array}$ & \\
\hline NO & & $\begin{array}{l}k_{3}=40(20) ; 32(14) \\
k_{1}=54(14)\end{array}$ & $k_{3}^{\text {th }}=32(14), k_{1}^{\text {th }}=54(14)$ \\
\hline $\mathrm{C}_{2} \mathrm{H}_{4}$ & $\mathrm{C}_{2} \mathrm{H}_{4}^{*}$ & $\begin{array}{l}k_{3}=56(21) \\
k_{1}=103(21)\end{array}$ & $\begin{array}{l}k_{3}^{\text {th }}=54(21) \\
k_{1}^{\text {th }}=103(21)\end{array}$ \\
\hline
\end{tabular}

\section{TABLEAU III}

$$
\mathrm{Ar}\left({ }^{3} \mathrm{P}_{1},{ }^{1} \mathrm{P}_{1}\right)+\mathrm{BC} \stackrel{k_{3,1}}{\rightarrow} \mathrm{Ar}+\mathrm{BC}^{*}\left(\mathrm{ou} \mathrm{B} \mathrm{B}^{*}+\mathrm{C}\right)
$$

(les références sont indiquées entre parenthèses après les valeurs des coefficients de collisions $k$ ) 
Le tableau III rassemble les résultats trouvés pour le transfert :

$\operatorname{Ar}\left({ }^{3} \mathrm{P}_{1},{ }^{1} \mathrm{P}_{1}\right)+\mathrm{BC} \stackrel{k_{3,1}}{\rightarrow} \mathrm{Ar}+\mathrm{BC}^{*}\left(\right.$ ou $\left.\mathrm{B}^{*}+\mathrm{C}\right)$

où $k_{3}$ est le coefficient de collision à partir de $\operatorname{Ar}\left({ }^{3} \mathrm{P}_{1}\right)$ et $k_{1}$ de $\operatorname{Ar}\left({ }^{1} \mathbf{P}_{1}\right)$.

Les valeurs calculées $k_{\text {th }}$ pour $\mathrm{NO}$ et $\mathrm{C}_{2} \mathrm{H}_{4}$ sont également indiquées dans le tableau III. L'accord avec les valeurs expérimentales est excellent. L'efficacité des transferts avec $\mathrm{C}_{2} \mathrm{H}_{4}$ s'explique par ce type d'interaction résonnante. En ce qui concerne NO, il est mentionné par McNeely [14] que l'accord entre les valeurs calculées et expérimentales peut être accidentel. En effet, la molécule NO dans son état fondamental n'a pas de moment angulaire nul et la section efficace de photoabsorption présente des singularités au voisinage de $\lambda=1048 \AA$ (variations d'un facteur 2 sur une bande de $4 \AA$ ).

Les transferts $\operatorname{Ar}\left({ }^{3} \mathbf{P}_{1},{ }^{1} \mathbf{P}_{1}\right)+\mathbf{H}_{2}$ sont étudiés en détail par Fink et al. [15]. Dans ce travail, les niveaux résonnants de l'argon sont photoexcités et le transfert sur $\mathrm{H}_{2}$ est analysé par les spectres de bandes de Lyman $\mathrm{H}_{2}\left(\mathrm{~B}^{1} \Sigma_{\mathrm{u}}^{+} \rightarrow \mathrm{x}^{1} \Sigma_{\mathrm{g}}^{+}\right)$. Les résultats expérimentaux sont en bon accord avec les valeurs calculées à partir de la théorie de Watanabe-Katsuura [12] lorsque l'écart énergétique entre les niveaux $\operatorname{Ar}\left({ }^{3} \mathrm{P}_{1}\right.$, $\left.{ }^{1} \mathrm{P}_{1}\right)$ et $\mathrm{H}_{2}\left(\mathrm{~B}^{1} \Sigma_{\mathrm{u}}^{+}, \mathrm{V}^{\prime}\right)$ ne dépasse pas $100 \mathrm{~cm}^{-1}$ environ (collisions résonnantes).

Pour les transferts sur l'azote, on trouve des écarts importants pour les valeurs de $k_{1}$ données dans les références [14] et [17]. De tels écarts s'observent également dans les transferts sur l'oxygène pour les valeurs de $k_{3}$ données dans les références [18] et [19]. Il est à remarquer que Bennet et al. [19] ont également étudié les transferts sur l'oxygène à partir des atomes métastables et résonnants du néon et du krypton. Les valeurs des coefficients de collisions sont dans le même ordre de grandeur. Velazco-Setser [22] ont comparé les transferts à partir des atomes métastables de l'argon et du xénon. Ils ont constaté qu'à l'exception de $\mathbf{H}_{2}$ et $\mathrm{N}_{2}$, les coefficients de collisions sont de 1 à 2 fois plus élevés avec le xénon. Il y aurait couplage des niveaux métastables $\left({ }^{3} \mathbf{P}_{2}\right)$ et résonnants $\left({ }^{3} \mathbf{P}_{1}\right)$ dans l'interaction avec $\mathrm{BC}$ et le transfert serait du type dipôle-dipôle. De fait, dans la relation (11), on a $k_{\mathrm{th}}(\mathrm{xe}) \sim 1,9 k_{\mathrm{th}}(\mathrm{Ar})$ calculé d'après les forces d'oscillateur $f\left(\mathrm{xe},{ }^{3} \mathrm{P}_{1}\right)=0,18$ [23] et $f\left(\mathrm{Ar},{ }^{3} \mathrm{P}_{1}\right)=0,061$ [24].

3.2 TRANSFERTS RÉACTIFS. - Ce type de transfert représenté par l'équation réactionnelle (1) se produit essentiellement avec les molécules halogénées $\mathrm{RX}$ où $\mathrm{X}=\mathrm{F}, \mathrm{Cl}$ et $\mathrm{Br}$. Le mécanisme est le suivant :

$$
\begin{aligned}
& \mathrm{A}^{*}+\mathrm{RX} \rightarrow \mathrm{AX}^{*}+\mathrm{R} \\
& \mathrm{AX}^{*} \rightarrow \mathrm{A}+\mathrm{X}+\mathrm{h} v .
\end{aligned}
$$

Le rayonnement émis à la suite de la réaction (13) est un fond continu dans l'U.V. proche.

$\mathrm{La}$ réaction (13) se produit avec les métastables de gaz rares $\mathrm{Ar}, \mathrm{Kr}$ et Xe. Les excimers formés $\mathrm{AX}^{*}$ se déexcitent vers un niveau fondamental répulsif, ce qui favorise des émissions lasers à rendement élevé. Ainsi, plusieurs types de lasers à fortes puissances $\left(10^{8}\right.$ à $10^{9}$ watts) fonctionnent à partir des excimers $\mathrm{ArF}^{*}, \mathrm{KrF}^{*}, \mathrm{XeF}^{*}$.

Certaines réactions de transfert sont connues. On trouve ainsi dans la référence [25] les réactions suivantes :

$$
\begin{aligned}
& \left.\mathrm{Xe}^{3}{ }^{3} \mathrm{P}_{2}\right)+\mathrm{Cl}_{2} \rightarrow \mathrm{XeCl}^{*}+\mathrm{Cl} \\
\text { avec } k= & 65 \times 10^{-11} \mathrm{~cm}^{3} \mathrm{~s}^{-1} \\
& \mathrm{Xe}\left({ }^{3} \mathrm{P}_{2}\right)+\mathrm{Br}_{2} \rightarrow \mathrm{XeBr}^{*}+\mathrm{Br} \\
\text { avec } k= & 62,4 \times 10^{-11} \mathrm{~cm}^{3} \mathrm{~s}^{-1}
\end{aligned}
$$

Les transferts réactifs (14) et (15) représentent la totalité de la réaction. Il n'en est pas de même avec d'autres produits halogénés comme $\mathrm{SOCl}_{2}, \mathrm{CF}_{3} \mathrm{I}$, $\mathrm{CF}_{3} \mathrm{OF}$ où il apparaît en plus divers produits de dissociation.

Gundel et al. [26] ont présenté une étude détaillée des transferts réactifs à partir des métastables de l'argon. Les résultats des réactions $\operatorname{Ar}\left({ }^{3} \mathrm{P}_{2,0}\right)+\mathrm{RX}$ avec $\mathrm{RX}=\mathrm{Cl}_{2}, \mathrm{NOCl}, \mathrm{CCl}_{4}, \mathrm{PCl}_{3}, \mathrm{HCl}, \mathrm{ClO}, \mathrm{Br}_{2}$ et des produits non halogénés OCS et $\mathrm{N}_{2} \mathrm{O}$ sont reportés dans le tableau IV. Les coefficients de collisions partiels $k_{i}$ indiqués dans ce tableau caractérisent les produits radiatifs seuls. La différence entre le coefficient de collision total $k_{\mathrm{t}}$ et $\sum_{i} k_{i}$ correspond donc aux autres transferts non radiatifs, l'effet Penning par exemple. Les coefficients $k_{i}$ sont déterminés à partir de spectres émis depuis le proche U.V., par les excimers $\mathrm{ArX}^{*}$, jusqu'au proche infrarouge, par les atomes $R^{*}$ ou $X^{*}$. Les flux de photons enregistrés au cours de ces réactions sont comparés à ceux émis à la suite du transfert $\operatorname{Ar}\left({ }^{3} \mathbf{P}_{2}\right)+\mathrm{Kr} \rightarrow \mathrm{Ar}+\mathrm{Kr}^{*}$.

Les rapports de branchement pour le niveau résonnant $\operatorname{Kr}\left(5 \mathrm{~s}[3 / 2]_{1}\right)$ (niveau supérieur de la raie de résonance $\lambda=1236 \AA)$ et le niveau $\operatorname{Kr}\left(5 \mathrm{p}[3 / 2]_{2}\right)$ (à l'origine de la raie $\lambda=7602 \AA$ ) sont connus. Ces raies spectrales servent ainsi à étalonner le spectre émis par les transferts réactifs.

On peut constater dans le tableau IV que le rendement en excimers est plus faible avec l'argon que le xénon. On passe de $100 \%$ dans la réaction (14) $\left(\mathrm{Xe}-\mathrm{Cl}_{2}\right)$ à environ $50 \%$ pour le mélange $\mathrm{Ar}-\mathrm{Cl}_{2}$.

Le spectre de bande émis par $\mathrm{ArCl}^{*}$ est situé entre 1650 et $2000 \AA$, il présente une structure vibrationnelle. Cette structure vibrationnelle est particulièrement développée si l'énergie de dissociation $D(\mathrm{RX})$ est faible. Il en résulte une diminution du rendement en excimers $\mathrm{ArX}^{*}$ au profit de produits dissociés $\mathrm{Ar}+\mathrm{X}^{*}$. C'est ce qu'on observe en passant de $\mathrm{Cl}_{2}$ à $\mathrm{NOCl}$ où $D\left(\mathrm{Cl}_{2}\right)=2,5 \mathrm{eV}$ et $D(\mathrm{NOCl})=1,6 \mathrm{eV}$.

Pour les autres molécules, les produits excités radiatifs ne représentent qu'une faible partie du transfert. Les réactions d'ionisation sont certainement importantes.

Avec $\mathrm{ClO}$, il est à noter que $\mathrm{ArO}^{*}$ n'a pas été détecté. 
TABLEAU IV

$$
\operatorname{Ar}\left({ }^{3} \mathbf{P}_{2,0}\right)+\mathrm{RX} \stackrel{k_{3}}{\rightarrow} \text { produits [26] }
$$

\begin{tabular}{|c|c|c|c|}
\hline Molécules RX & Produits formés & $\begin{array}{c}\text { Coefficients } \\
\text { de collisions partiels } \\
k_{\mathrm{i}}\left(10^{-11} \mathrm{~cm}^{3} \mathrm{~s}^{-1}\right)\end{array}$ & $\begin{array}{c}\text { Coefficients } \\
\text { de collisions } \\
\text { total } \\
k_{\mathrm{t}}\left(10^{-11} \mathrm{~cm}^{3} \mathrm{~s}^{-1}\right)\end{array}$ \\
\hline Molécules & & 135 & - \\
\hline $\begin{array}{l}\text { Molecules } \\
\text { halogénées } \\
\qquad \mathrm{Cl}_{2}\end{array}$ & $\begin{array}{l}\mathrm{ArCl}+\mathrm{Cl} \\
\mathrm{ArCl}+\mathrm{Cl}^{*} \\
\mathrm{Ar}+\mathrm{Cl}_{2}^{*}\end{array}$ & $\begin{array}{l}35 \\
11 \\
1,8\end{array}$ & 71 \\
\hline $\mathrm{NOCl}$ & $\begin{array}{l}\mathrm{ArCl}^{*}+\mathrm{NO} \\
\mathrm{Ar}+\mathrm{NO}+\mathrm{Cl}^{*} \\
\mathrm{Ar}+\mathrm{Cl}+\mathrm{NO}^{*}\end{array}$ & $\mid \begin{array}{r}9,3 \\
30,5 \\
6\end{array}$ & 48 \\
\hline $\mathrm{CCl}_{4}$ & $\mid \begin{array}{l}\mathrm{ArCl}^{*}+\mathrm{CCl}_{3} \\
\mathrm{CCl}_{3}^{*}+\mathrm{Cl}+\mathrm{Ar}\end{array}$ & $\begin{array}{l}5,7 \\
1,2\end{array}$ & 100 \\
\hline $\mathrm{PCl}_{3}$ & $\begin{array}{l}\mathrm{ArCl}^{*}+\mathrm{PCl}_{2} \\
\mathrm{PCl}_{2}^{*}+\mathrm{Cl}+\mathrm{Ar}\end{array}$ & $\begin{array}{l}0,25 \\
0,5\end{array}$ & 53 \\
\hline $\mathrm{HCl}$ & $\mid \begin{array}{l}\mathrm{ArCl}^{*}+\mathrm{H} \\
\mathrm{HCl}^{*}+\mathrm{Ar}\end{array}$ & $\mid \begin{array}{l}0,49 \\
0,63\end{array}$ & 35 \\
\hline $\mathrm{ClO}$ & $\mid \begin{array}{l}\mathrm{ArCl}^{*}+\mathrm{O} \\
\mathrm{Cl}^{*}+\mathrm{O}\end{array}$ & $\mid \begin{array}{l}13 \\
0,2\end{array}$ & 38 \\
\hline $\mathrm{Br}_{2}$ & $\begin{array}{l}\mathrm{ArBr}^{*}+\mathrm{Br} \\
\mathrm{Br}^{*}+\mathrm{Br}+\mathrm{Ar}\end{array}$ & $\mid \begin{array}{c}0,52 \\
64,7\end{array}$ & 66 \\
\hline $\begin{array}{l}\text { Molécules } \\
\text { non halogénées } \\
\qquad \mathrm{N}_{2} \mathrm{O}\end{array}$ & $\begin{array}{l}\mathrm{N}_{2}^{*}+\mathrm{O}+\mathrm{Ar} \\
\mathrm{NO}^{*}+\mathrm{N}+\mathrm{Ar} \\
\mathrm{O}^{*}+\mathrm{N}_{2}+\mathrm{Ar} \\
\mathrm{CO}^{*}+\mathrm{S}+\mathrm{Ar}\end{array}$ & $\begin{array}{l}50 \\
1,4 \\
0,086 \\
2,85\end{array}$ & 52 \\
\hline OCS & $\begin{array}{l}\mathrm{CS}^{*}+\mathrm{O}+\mathrm{Ar} \\
\mathrm{S}^{*}+\mathrm{CO}+\mathrm{Ar}\end{array}$ & $\begin{array}{r}0,2 \\
0,34\end{array}$ & 79 \\
\hline
\end{tabular}

Molécules RF

$\mathrm{F}_{2}$

$\mathrm{OF}_{2}$

$\mathrm{CF}_{3} \mathrm{OF}$

$\mathrm{NF}_{3}$

$\mathrm{N}_{2} \mathrm{~F}_{4}$

$\mathrm{NOF}$
TABLEAU $\mathrm{V}$

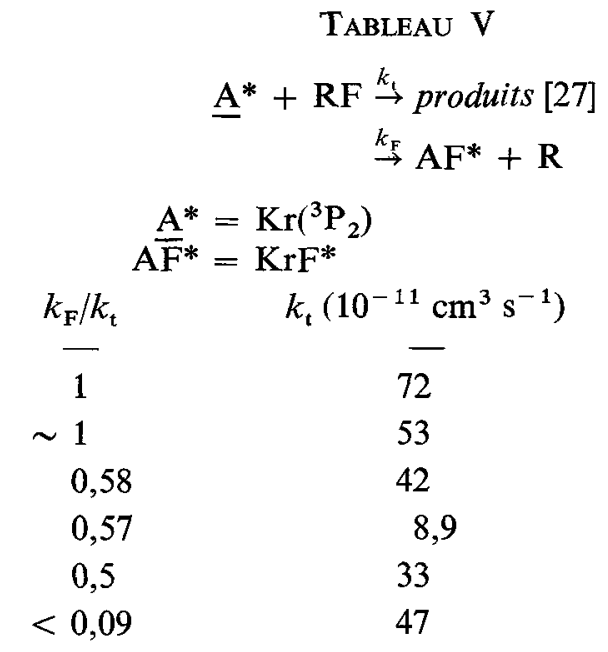$$
\stackrel{k_{F}}{\rightarrow} \mathrm{AF}^{*}+\mathrm{R}
$$

Coefficients

collisions collisions partiels
71

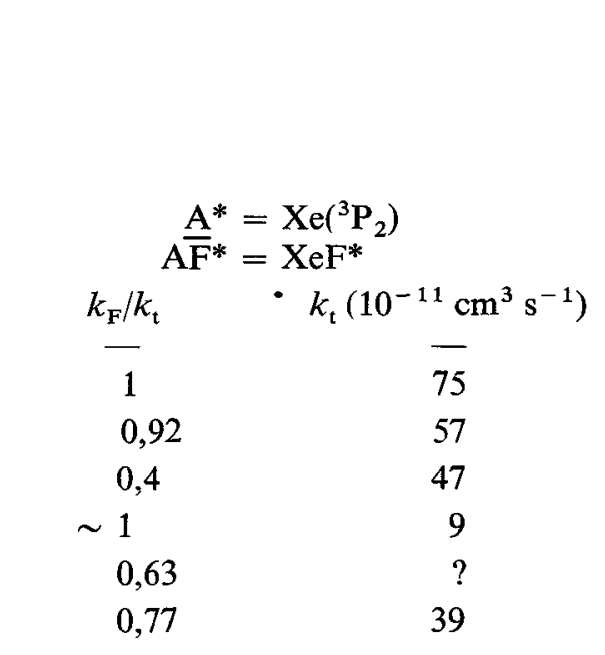

Avec $\mathrm{Br}_{2}$ et $\mathrm{N}_{2} \mathrm{O}$, on constate que la majeure partie $\mathrm{du}$ transfert est dissociative. Pour $\mathrm{N}_{2} \mathrm{O}$, l'azote est excité principalement sur les bas niveaux vibrationnels de l'état $\mathbf{B}^{3} \Pi_{\mathrm{g}}$.

L'effet Penning disparait progressivement avec les atomes métastables du krypton et du xénon, et la production en excimers est élevée. Les meilleurs rendements ont été obtenus avec des produits fluorés, comme le montre le tableau $V$ tiré de la référence [27].

L'excimer formé à la suite d'un transfert réactif présente un caractère ionique marqué suivant la polarité $\left(\mathrm{A}^{+} \mathrm{X}^{-}\right)$. Les produits halogénés possèdent en effet une grande affinité électronique $(0,5-2 \mathrm{eV})$ ce qui produit une intersection entre le potentiel 
d'entrée $A^{*}+R X$ et la courbe coulombienne $\mathrm{A}^{+}+\mathrm{RX}^{-}$à grandes distances internucléaires $(R>4 \AA)$. Si l'énergie de dissociation de $\mathrm{RX}$ est inférieure à celle de l'excimer $\mathrm{AX}^{*}$, le radical $R$ est éjecté et les interactions se font suivant la chaîne :

$$
\begin{aligned}
\left(\mathrm{A}^{*}+\mathrm{RX}\right) \rightarrow\left(\mathrm{A}^{+}-\mathrm{RX}^{-}\right)+\left(\mathrm{A}^{+} \mathrm{X}^{-}\right) & +R \rightarrow \\
& \rightarrow \mathrm{AX}^{*}+\mathrm{R} .
\end{aligned}
$$

Par ailleurs, à l'exemple de la réaction

$$
\operatorname{Ar}\left({ }^{3} \mathrm{P}_{2}\right)+\mathrm{NOCl} \text {, }
$$

il ne faut pas que l'énergie de dissociation de RX soit trop faible sinon l'excimer $\mathrm{AX}^{*}$ est formé dans un état vibrationnel très excité et se dissocie en $\mathrm{A}+\mathrm{X}^{*}$. De bonnes valeurs pour $D(\mathrm{RX})$ se situent entre 2 et $4 \mathrm{eV}$. En général, les transferts $\left(\mathrm{A}^{+} \mathrm{X}^{-}\right) \rightarrow \mathrm{AX}^{*}$ sont directs pour les halogènes diatomiques $\left(\mathrm{F}_{2}, \mathrm{Cl}_{2}\right.$, $\mathrm{FCl}$ ). Dans le cas de molécules multi-halogénées, le complexe $\left(\mathrm{A}^{+} \mathrm{X}^{-}\right)$a une longue durée de vie et conduit aux espèces $\mathrm{RX}^{*}$.

Des transferts réactifs se produisent avec des espèces métastables autres que les gaz rares à l'exemple des réactions $\mathrm{N}\left({ }^{2} \mathrm{D}\right)+\mathrm{N}_{2}, \mathrm{O}_{2}, \mathrm{NO}, \mathrm{N}_{2} \mathrm{O}, \mathrm{CO}_{2}$ étudiées par Lin-Kaufman [28].

Ces réactions sont d'un grand intérêt en chimie des plasmas. Avec $\mathrm{O}_{2}$, la réaction s'écrit :

$$
\mathrm{N}\left({ }^{2} \mathrm{D}\right)+\mathrm{O}_{2} \stackrel{k_{1}}{\rightarrow} \mathrm{NO}+\mathrm{O} .
$$

L'oxyde d'azote est rapidement détruit par l'azote atomique à l'état fondamental $\mathrm{N}\left({ }^{4} \mathrm{~S}\right)$, suivant :

$$
\mathrm{NO}+\mathrm{N}\left({ }^{4} \mathrm{~S}\right) \stackrel{k_{2}}{\rightarrow} \mathrm{N}_{2}+\mathrm{O}
$$

avec

$$
k_{1}=7 \times 10^{-12} \mathrm{~cm}^{3} \mathrm{~s}^{-1}, k_{2}=3 \times 10^{-11} \mathrm{~cm}^{3} \mathrm{~s}^{-1} \text {. }
$$

\section{TABLEAU VI}

$$
\mathrm{N}\left({ }^{2} \mathrm{D}\right)+\mathrm{BC} \stackrel{k}{\rightarrow} \text { produits }[28]
$$

$\begin{array}{cccccc}\text { Molécules BC } & \mathrm{N}_{2} & \mathrm{O}_{2} & \mathrm{NO} & \mathrm{N}_{2} \mathrm{O} & \mathrm{CO}_{2} \\ - & - & - & - & - & - \\ k\left(10^{-12} \mathrm{~cm}^{3} \mathrm{~s}^{-1}\right) & 0,016 & 6 \pm 2 & 70 \pm 25 & 3,5 \pm 1,2 & 0,5 \pm 0,2\end{array}$

Le résultat des réactions (16) et (17) est de produire deux atomes d'oxygène par atome métastable $\mathrm{N}\left({ }^{2} \mathrm{D}\right)$. Les coefficients de collisions pour les autres molécules $\mathrm{BC}$ sont indiqués dans le tableau VI. Bien que les valeurs de ces coefficients ne soient pas élevées, l'intérêt de telles réactions peut provenir des fortes concentrations d'espèces $\mathrm{N}\left({ }^{2} \mathrm{D}\right)$ obtenues dans les décharges, bien supérieures à celles des atomes métastables des gaz rares.

4. Transferts entre états excités de l'azote. - Nous considérons les phénomènes de relaxation des espèces excitées de l'azote dans l'azote pur. Nous nous intéressons en particulier aux transferts d'excitation qui participent à la relaxation globale d'un niveau excité donné. Ces phénomènes de transferts ont pour beaucoup été étudiés dans des post-décharges où la réassociation de l'azote atomique produit une postluminescence caractéristique.

4.1 Ré-association de l'azote atomique. - En post-décharge, le mécanisme de ré-association de l'azote atomique est représenté par l'équation réactionnelle suivante :

$$
\begin{aligned}
\mathrm{N}+\mathrm{N}+\mathrm{N}_{2} \rightarrow \mathrm{N}_{2}\left(\mathrm{~B}^{3} \Pi_{\mathrm{g}}, 9<v^{\prime}<12\right)+\mathrm{N}_{2} \\
\mathrm{~N}_{2}\left(\mathrm{~B}^{3} \Pi_{\mathrm{g}}, 9<v^{\prime}<12\right) \rightarrow \mathrm{N}_{2}\left(\mathrm{~A}^{3} \Sigma_{\mathrm{u}}^{+}, v^{\prime \prime}\right)+\mathrm{h} v \\
\left(1^{\mathrm{er}}\right. \text { système positif). }
\end{aligned}
$$

L'émission du $1^{\text {er }}$ système positif à partir des niveaux vibrationnels élevés de l'état $\mathrm{B}^{3} \Pi_{\mathrm{g}}$ est ainsi caractéristique de ce phénomène. L'analyse fine de la réaction (18) fait l'objet de controverses. Ainsi, suivant Becker et al. [29] l'état $\mathrm{N}_{2}\left({ }^{5} \Sigma_{\mathrm{g}}^{+}\right)$sert d'intermédiaire :

$$
\begin{aligned}
& \mathrm{N}+\mathrm{N} \rightarrow\left[\mathrm{N}_{2}\left({ }^{5} \Sigma_{\mathrm{g}}^{+}\right)\right] \rightarrow\left[\mathrm{N}_{2}\left(\mathrm{~B}^{3} \Pi_{\mathrm{g}}, v^{\prime}=13\right)\right] \\
& {\left[\mathrm{N}_{2}\left(\mathrm{~B}, v^{\prime}=13\right)\right]+\mathrm{N}_{2} \rightarrow \mathrm{N}_{2}(\mathrm{~B}, 9<v<12)+\mathrm{N}_{2} .}
\end{aligned}
$$

Les crochets désignent des états dissociatifs. D'après Shemansky [30], la réaction (19) est trop lente et ne rend pas compte de toute la distribution vibrationnelle de $N_{2}\left(B^{3} \Pi_{\mathrm{g}}\right)$. Les mécanismes suivants sont proposés :

$$
\begin{aligned}
& \mathrm{N}+\mathrm{N}+\mathrm{N}_{2} \rightarrow \mathrm{N}_{2}\left(\mathrm{~A}^{3} \Sigma_{\mathrm{u}}^{+}, v^{\prime}\right)+\mathrm{N}_{2} \\
& \mathrm{~N}_{2}\left(\mathrm{~A}, v^{\prime}\right)+\mathrm{N}_{2} \rightarrow \mathrm{N}_{2}\left(\mathrm{~B}, v^{\prime \prime}\right)+\mathrm{N}_{2} .
\end{aligned}
$$

La réaction (21) représente la plus grande part de la relaxation des niveaux $\mathrm{N}_{2}\left(\mathrm{~A}, v^{\prime}>7\right)$ dont nous donnons les coefficients de relaxation $k_{\mathrm{O}}^{\mathrm{A}}\left(v^{\prime}\right)$, avec ceux de $k_{\mathrm{Q}}^{\mathrm{B}}\left(v^{\prime \prime}\right)$ de $\mathrm{B}^{3} \Pi_{\mathrm{g}}$, dans le tableau VII. Les niveaux $v^{\prime}$ de $\mathrm{A}^{3} \Sigma_{\mathrm{u}}^{+}$proches de $v^{\prime \prime}$ de $\mathrm{B}^{3} \Pi_{g}$ sont également indiqués dans le tableau VII.

4.2 Transferts D'EXCitation a PartiR DE $\mathrm{N}_{2}\left(\mathrm{~B}^{3} \Pi_{\mathrm{g}}\right)$. - La relaxation de $\mathrm{N}_{2}\left(\mathrm{~B}^{3} \Pi_{\mathrm{g}}, v\right)$ dont les coefficients $k_{\mathrm{Q}}^{\mathrm{B}}(v)$ sont donnés dans le tableau VII peut alimenter plusieurs états électroniques de l'azote. Les mécanismes suivants ont été proposés :

a) Echanges $\mathrm{N}_{2}\left(\mathrm{~B}^{3} \Pi_{\mathrm{g}}\right) \rightleftarrows \mathrm{N}_{2}\left(\mathrm{~A}^{3} \Sigma_{\mathrm{u}}^{+}\right)$

$$
\begin{aligned}
& \mathrm{N}_{2}\left(\mathrm{~B}, v_{1}>7\right)+\mathrm{N}_{2} \rightarrow \mathrm{N}_{2}\left(\mathrm{~A}, v_{2}\right)+\mathrm{N}_{2} \\
& \mathrm{~N}_{2}\left(\mathrm{~A}, v_{2}\right)+\mathrm{N}_{2} \rightarrow \mathrm{N}_{2}\left(\mathrm{~B}, v_{1}-1\right)+\mathrm{N}_{2} .
\end{aligned}
$$

Il s'agit d'échanges mutuels indiqués par Young [31] sans données quantitatives. La réaction (21) constitue une partie de ces échanges.

b) Echanges $\mathrm{N}_{2}\left(\mathrm{~B}^{3} \Pi_{\mathrm{g}}\right) \rightleftarrows \mathrm{N}_{2}\left(\mathrm{~W}^{3} \Delta_{\mathrm{u}}\right)$

Les transferts sont radiatifs (réf. [32]) :

$$
\begin{aligned}
& \mathrm{N}_{2}(\mathrm{~B}, v=6) \rightarrow \mathrm{N}_{2}(\mathrm{~W}, v=4)+\mathrm{h} v \\
& \mathrm{~N}_{2}(\mathrm{~W}, v=4) \rightarrow \mathrm{N}_{2}(\mathrm{~B}, v=1)+\mathrm{h} v .
\end{aligned}
$$




\section{TABLEAU VII}

$$
\begin{aligned}
& N_{2}\left(\mathrm{~A}, v^{\prime}\right)+\mathrm{N}_{2} \stackrel{k_{(\mathrm{A}}^{\mathrm{A}\left(v^{\prime}\right)} \rightarrow}{\rightarrow} \text { produits }[30] \\
& N_{2}\left(\mathrm{~B}, v^{\prime \prime}\right)+\mathrm{N}_{2} \stackrel{k_{\left(v^{\prime}\right.}\left(v^{\prime \prime}\right)}{\rightarrow} \text { produits }[30]
\end{aligned}
$$

\begin{tabular}{|c|c|c|c|c|c|c|c|c|c|c|c|c|}
\hline $\mathrm{B}^{3} \Pi_{\mathrm{g}}, v^{\prime \prime}$ & 0 & 1 & 2 & 3 & 4 & 5 & 6 & 7 & 8 & 9 & 10 & 11 \\
\hline $\mathrm{A}^{3} \Sigma_{\mathrm{u}}^{+}, v^{\prime}$ & 7 & 8 & $9-10$ & 11 & $\begin{array}{l}12 \\
13\end{array}$ & 14 & 16 & 17 & 19 & 21 & $\begin{array}{l}22 \\
23\end{array}$ & $\begin{array}{l}24 \\
25\end{array}$ \\
\hline$k_{\mathrm{Q}}^{\mathrm{A}}\left(v^{\prime}\right) 10^{-11} \mathrm{~cm}^{3} \mathrm{~s}^{-1}$ & & & 1 & 8,8 & 2 & 2 & 4 & 5 & 1,9 & 3 & 3,5 & 4,5 \\
\hline$k_{\mathrm{Q}}^{\mathbf{B}}\left(v^{\prime \prime}\right) 10^{-11} \mathrm{~cm}^{3} \mathrm{~s}^{-1}$ & $\sim 1$ & $\sim 1$ & 1,9 & 1,7 & 1,8 & 2,3 & 4,1 & 6,6 & 7,2 & 9,8 & 6,3 & 2 \\
\hline
\end{tabular}

Dans le niveau $v=0$, les états $\mathrm{B}$ et $\mathrm{W}$ sont couplés et relaxent vers l'état $A^{3} \Sigma_{u}^{+}$(réf. [33]).

c) Echanges $\mathrm{N}_{2}\left(\mathrm{~B}^{3} \Pi_{\mathrm{g}}\right) \rightleftarrows \mathrm{N}_{2}\left(\mathrm{~B}^{3} \Sigma_{\mathrm{u}}^{-}\right)$

Gartner et Thrush [34] indiquent le couplage suivant :

$$
\mathrm{N}_{2}\left(\mathrm{~B}^{\prime 3} \Sigma_{\mathrm{u}}^{-}, v^{\prime}\right)+\mathrm{N}_{2} \rightleftarrows \mathrm{N}_{2}\left(\mathrm{~B}^{3} \Pi_{\mathrm{g}}, v^{\prime}+4\right)+\mathrm{N}_{2} .
$$

Les réactions à partir du niveau $\mathrm{B}^{3} \Pi_{\mathrm{g}}, v^{\prime \prime}=11$ ont été étudiées en particulier :

$\mathrm{N}_{2}(\mathrm{~B}, 11)+\mathrm{N}_{2} \stackrel{k_{11}}{\rightarrow} \mathrm{N}_{2}\left(\mathrm{~B}^{\prime}, 7\right)+\mathrm{N}_{2}$

$\mathrm{N}_{2}\left(\mathrm{~B}^{\prime}, 7\right)+\mathrm{N}_{2} \stackrel{k_{0}^{7}}{\rightarrow}$ produits, surtout $\mathrm{N}_{2}(\mathrm{~B})$

avec $k_{11}=4,7 \times 10^{-12} \mathrm{~cm}^{3} \mathrm{~s}^{-1}$ et

$$
k_{\mathrm{Q}}^{7}=6 \times 10^{-12} \mathrm{~cm}^{3} \mathrm{~s}^{-1} \text {. }
$$

4.3 ACtion DE L'AZOTE ATOMiQue. - L'azote atomique désexcite les états $\mathrm{N}_{2}\left(\mathrm{~B}^{3} \Pi_{\mathrm{g}}\right)$ suivant la réaction (réf. [35]) :

$$
\mathrm{N}_{2}\left(\mathrm{~B}^{3} \Pi_{\mathrm{g}}\right)+\mathrm{N}\left({ }^{4} \mathrm{~S}\right) \stackrel{k_{\mathrm{B}}^{\mathrm{B}}}{\rightarrow} \mathrm{N}_{2}\left(\mathrm{a}^{1} \Pi_{\mathrm{g}}, \mathrm{a}^{1} \Sigma_{\mathrm{u}}^{-}\right)+\mathrm{N}\left({ }^{4} \mathrm{~S}\right)
$$

avec $k_{\mathrm{N}}^{\mathrm{B}}=3,4 \times 10^{-11} \mathrm{~cm}^{3} \mathrm{~s}^{-1}$.

Cette action de l'azote atomique se produit également sur d'autres états excités de l'azote. Ainsi, pour les molécules métastables $\mathrm{N}_{2}\left(\mathrm{~A}^{3} \Sigma_{\mathrm{u}}^{+}\right)$nous avons (réf. [36]) :

$$
\begin{aligned}
& \mathrm{N}_{2}\left(\mathrm{~A}^{3} \Sigma_{\mathrm{u}}^{+}\right)+\mathrm{N}\left({ }^{4} \mathrm{~S}\right) \stackrel{k_{\hat{N}}^{A}}{\rightarrow} \mathrm{N}_{2}\left(\mathrm{X}^{1} \Sigma_{\mathrm{g}}^{+}\right)+\mathrm{N}\left({ }^{2} \mathrm{P}\right) \\
& k_{\mathrm{N}}^{\mathrm{A}}=5 \times 10^{-11} \mathrm{~cm}^{3} \mathrm{~s}^{-1} .
\end{aligned}
$$

L'azote atomique $\mathrm{N}\left({ }^{2} \mathrm{P}\right)$ produit par la réaction (27) se désexcite radiativement :

$$
\begin{aligned}
\mathrm{N}\left({ }^{2} \mathrm{P}\right) & \rightarrow \mathrm{N}\left({ }^{2} \mathrm{D}\right)+\mathrm{h} v(\lambda=10400 \AA) \\
& \rightarrow \mathrm{N}\left({ }^{4} \mathrm{~S}\right)+\mathrm{h} v(\lambda=3466 \AA) .
\end{aligned}
$$

Une possibilité de désexcitation de l'azote atomique métastable $\mathrm{N}\left({ }^{2} \mathrm{D}\right)$ est donnée par la réaction suivante (réf. [37]) :

$$
\mathrm{N}\left({ }^{2} \mathrm{D}\right)+\mathrm{N}\left({ }^{4} \mathrm{~S}\right) \rightarrow \mathrm{N}_{2}\left(\mathrm{C}^{3} \Pi_{\mathrm{u}}, v^{\prime}=3,4\right) .
$$

La réaction (28) est alors caractérisée par l'émission $\mathrm{du} 2^{\mathrm{e}}$ système positif à partir des niveaux vibrationnels élevés :

$$
\mathrm{N}_{2}\left(\mathrm{C}, v^{\prime}=3 \text { et } 4\right) \rightarrow \mathrm{N}_{2}\left(\mathrm{~B}^{3} \Pi_{\mathrm{g}}, v^{\prime \prime}\right)+\mathrm{h} v .
$$

4.4 TRANSFERTS D'EXCITATION A PARTIR DE $\mathrm{N}_{2}\left(\mathrm{~A}^{3} \Sigma_{\mathrm{u}}^{+}, \mathrm{E}^{3} \Sigma_{\mathrm{g}}^{+}, \mathrm{a}^{1} \Pi_{\mathrm{g}}\right)$. - Les molécules métastables $\mathrm{N}_{2}\left(\mathrm{~A}^{3} \Sigma_{\mathrm{u}}^{+}\right)$s'auto-désexcitent suivant (réf. [38] :

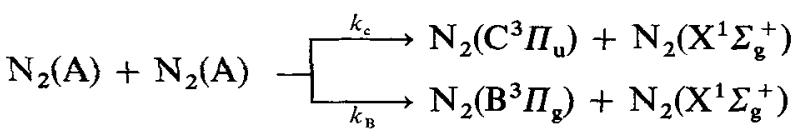

avec $k_{\mathrm{c}}=2,5 \times 10^{-10} \mathrm{~cm}^{3} \mathrm{~s}^{-1}$,

$$
k_{\mathrm{B}}=1,1 \times 10^{-9} \mathrm{~cm}^{3} \mathrm{~s}^{-1} .
$$

La réaction (29) a pour effet de surpeupler les niveaux vibrationnels élevés de l'état $C^{3} \Pi_{\mathrm{u}}$ (réf. [38] [39]). Ce phénomène rentre ainsi en compétition avec celui représenté par la réaction (28).

Les molécules métastables $\mathrm{N}_{2}\left(\mathrm{E}^{3} \Sigma_{\mathrm{g}}^{+}\right)$se désexcitent au profit de $\mathrm{C}^{3} \Pi_{\mathrm{u}}$, suivant la réaction :

$$
\mathrm{N}_{2}(\mathrm{E})+\mathrm{N}_{2}(\mathrm{X}) \stackrel{k_{\mathrm{E}}}{\rightarrow} \mathrm{N}_{2}(\mathrm{C})+\mathrm{N}_{2}(\mathrm{X})
$$

avec $k_{\mathrm{E}}=5,4 \times 10^{-11} \mathrm{~cm}^{3} \mathrm{~s}^{-1}$, ce qui représente la moitié du coefficient de relaxation global du niveau $\mathrm{E}^{3} \Sigma_{\mathrm{g}}^{+}$(réf. [40]).

Les espèces $\mathrm{N}_{2}\left(\mathrm{a}^{1} \Pi_{\mathrm{g}}\right)$ alimentent l'état $\mathrm{B}^{3} \Pi_{\mathrm{g}}$ suivant la réaction (réf. [41]) :

$$
\mathrm{N}_{2}(\mathrm{a})+\mathrm{N}_{2}(X) \rightarrow \mathrm{N}_{2}(\mathrm{~B})+\mathrm{N}_{2}(X)
$$

5. Conclusion. - L'excitation de l'azote dans les mélanges $A r-\mathrm{N}_{2}$ constitue une synthèse d'un bon nombre des mécanismes précédemment décrits. Nous avons repris dans le tableau VIII le schéma cinétique donné par Hill [10] en le complétant avec les données récentes de Shemansky [30] et Burns [40]. Dans ce schéma cinétique, Hill indique une proportion de $60 \%$ du transfert $\underline{A r}^{*}+N_{2}$ pour l'état $N_{2}\left(B^{3} \Pi_{g}\right)$. 


\section{TABLEAU VIII}

Schéma cinétique relatif à l'excitation de $\mathrm{N}_{2}$ à la suite du transfert argon métastable-azote Les coefficients de collisions $k$ sont en $10^{-11} \mathrm{~cm}^{3} \mathrm{~s}^{-1}$

Kinetic diagram for the $\mathrm{N}_{2}$ excitation due to the metastable argon-nitrogen transfer The $k$-rate coefficients are in $10^{-11} \mathrm{~cm}^{3} \mathrm{~s}^{-1}$

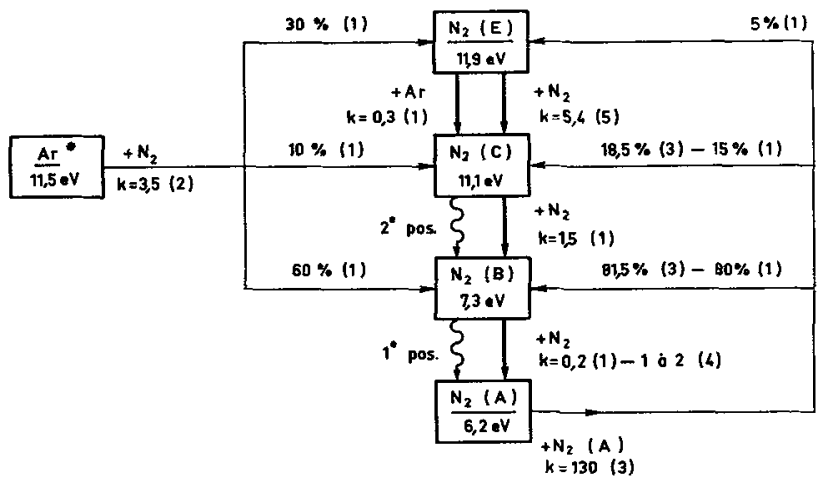

[1] HILl et al. (1974) réf. [10].

[2] Setser-Stedman (1970) réf. [9].

[3] HaYs-OSKaM (1973) réf. [38].

[4] Shemansky (1976) réf. [30].

[5] BuRNs et al. (1976) réf. [40].

Nous avons vu au paragraphe 3.1 que ce chiffre est certainement trop fort. De même, on ne comprend pas pourquoi $30 \%$ du transfert alimente l'état $\mathrm{N}_{2}\left(\mathrm{E}^{3} \Sigma_{\mathrm{g}}^{+}\right)$puisque l'énergie des atomes métastables et résonnants de la configuration $3 \mathrm{p}^{5} 4 \mathrm{~s}$ de l'argon est située entre 11,5 et $11,8 \mathrm{eV}$. Pour que l'état $\mathrm{N}_{2}(\mathrm{E})$ soit peuplé, il faut que l'état fondamental $\mathrm{N}_{2}\left(\mathrm{X}^{1} \Sigma_{\mathrm{g}}^{+}\right)$ soit vibrationnellement très excité. En ce qui concerne les transferts $\mathrm{N}_{2}(\mathrm{~A})+\mathrm{N}_{2}(\mathrm{~A})$, indiqués au paragraphe 4.4 , Hill suppose que $10 \%$ de la réaction alimente l'état $\mathrm{N}_{2}(\mathrm{E})$, ce qui lui permet de boucler le schéma cinétique. Une des conséquences d'un tel schéma, avec les données d'avant 1974 , est de prévoir une inversion de population entre les niveaux $\mathrm{N}_{2}\left(\mathrm{C}^{3} \Pi_{\mathrm{u}}, v^{\prime}=0\right)$ et $\mathrm{N}_{2}\left(\mathrm{~B}^{3} \Pi_{\mathrm{g}}, v^{\prime \prime}=1\right)$, raie

$$
\lambda=3577 \AA \text {, }
$$

dans les décharges de faisceaux d'électrons pulsés (réf. [10]). L'inversion de population $\mathrm{N}_{2}\left(\mathrm{C}, v^{\prime}=0\right.$ ) et $\mathrm{N}_{2}\left(\mathrm{~B}, v^{\prime \prime}=0\right)$, raie $\lambda=3371 \AA$, n'est pas obtenue. En fait, l'émission laser de la raie $3371 \AA$ a été obtenue par Puech [42] qui a montré qu'un tel résultat était possible si le transfert $\mathrm{Ar}^{*}+\mathrm{N}_{2}$ n'alimentait pas l'état $\mathrm{B}^{3} \Pi_{\mathrm{g}}$. Il s'agit d'une nouvelle preuve du respect de la quasi-résonance au profit de l'état $\mathrm{N}_{2}\left(\mathrm{C}^{3} \Pi_{\mathrm{u}}\right)$ dans les transferts $\mathrm{Ar}^{*}-\mathrm{N}_{2}$.

\section{Bibliographie}

[1] Delcroix, J. L., Matos-Ferreira, C. et Ricard, A., Atomes et molécules métastables dans les gaz ionisés. Chap. 5 (Editions du C.N.R.S.) 1975.

[2] West, W. P. et al., J. Chem. Phys. 63 (1975) 1237.

[3] Howard, J. S. et al., J. Phys. B 6 (1973) L 109.

[4] Riola, J. P. et al., J. Phys. B 7 (1974) 376.

[5] Lindinger, W., Schmeltekopf, A. L. et Fehsenfeld, F. C., J. Chem. Phys. 61 (1974) 2890.

[6] Coxon, J. A., Clyne, M. A. A., Setser, D. W., Chem. Phys. 7 (1975) 255.

[7] Lee, W., Martin, R., J. Chem. Phys. 63 (1975) 962.

[8] Sanders, R. A. et al., J. Chem. Phys. 65 (1976) 2700.

[9] Setser, D. W., Stedman, D. H., J. Chem. Phys. $\mathbf{5 3}$ (1970) 1004.

[10] HILL, R. M. et al., Report S.R.I. no MP 74-39 (1974).

[11] Touzeav, M. et al., J. Physique Colloq. 38 (1977) C3.

[12] Watanabe, T., Katsuura, K., J. Chem. Phys. 47 (1967) 800.

[13] Klots, C. E., ANderson, V. E., J. Chem. Phys. 56 (1972) 124.

[14] MCNeely, J. R. et al., J. Chem. Phys. 63 (1975) 2717.

[15] Fink, E. H. et al., J. Chem. Phys. 56 (1972) 3608.

[16] Bourene, M. et al., J. Chem. Phys. 63 (1975) 1668.

[17] Deschamps, J., Rrcard, A., J. Physique C 2 (1973) 123.

[18] Clark, I. D., Masson, A. J., Wayne, R. P., Mol. Phys. 23 (1972) 995 .

[19] BENNET, W. R., Jr. Gaseous Optical Masers, Quantum Electronics III (Columbia Univ. Press, N.Y.) 1963.

[20] Chapman, C. J., Masson, A. J., Wayne, R. P., Mol. Phys. 23 (1972) 979.

[21] Hurst, G. S., Wagner, E. B., Payne, M. G., J. Chem. Phys. 61 (1974) 3680.
[22] Velazco, J. E., Setser, D. W., Chem. Phys. Lett. 25 (1974) 197.

[23] WIEME, W., MORTIER, P., Physica 65 (1973) 198.

[24] Wirse, W. L. et al., Atomic Transition Probabilities. Vol. 2 (N.S.R.D.S.-N.B.S.) (1969) 22.

[25] Velazco, J. E., Setser, D. W., J. Chem. Phys. 62 (1975) 1990.

[26] Gundel, L. A. et al., J. Chem. Phys. 64 (1976) 4390.

[27] Velazco, J. E., Kolts, J. H., Setser, D. W., J. Chem. Phys. 65 (1976) 3468.

[28] Lin, C. L., Kaufman, F., J. Chem. Phys. 55 (1971) 3760.

[29] Becker, K. F. et al., Faraday Disc. Chem. Soc. 53 (1972) 35.

[30] Shemansky, D. E., J. Chem. Phys. 64 (1976) 565.

[31] Young, S. J., J. Chem. Phys. 60 (1974) 5050.

[32] Benesch, W. M., SAUM, K. A., J. Phys. B 4 (1971) 732.

[33] HeIDNeR, R. F. et al., Aerospace Report ATR-75 (1975).

[34] Gartner, E. M., Thrush, B. A., Proc. Roy. Soc. London A 346 (1975) 103.

[35] Colde, M. F., Thrush, B. A., Faraday Disc. Chem. Soc. 53 (1972) 52.

[36] Young, R. A., St. John, G. A., J. Chem. Phys. 48 (1968) 895.

[37] Tanaka, Y., Le Blanc, F., Jursa, A., J. Chem. Phys. 30 (1959) 1624.

[38] Hays, G. N., Oskam, H. J., J. Chem. Phys. 59 (1973) 1507.

[39] Stedman, D. H., Setser, D. W., J. Chem. Phys. 50 (1969) 2256.

[40] Burns, D. J., Golden, D. E., Galliardt, D. W., J. Chem. Phys. 65 (1976) 2616.

[41] Ravodina, O. V., Popova, T. N., Opt. Spectry 38 (1975) 145.

[42] PuEch, V., Thèse $n^{o} 2061$, Orsay (1976). 